\title{
BIO.I 7 - Construction of a scFv library using directed evolution for rituximab- based therapies: using phage display towards antibody affinity maturation
}

\author{
Marcus Rafael Lobo Bezerra ${ }^{1 *}$; Andrea Queiroz Maranhao ${ }^{2}$; Igor Cabral Studart ${ }^{1}$; Larissa \\ Queiroz Pontes ${ }^{1}$; Marcela Helena Gambim Fonseca ${ }^{3}$; Gilvan Pessoa Furtado ${ }^{3}$. \\ 1UFCe - Universidade Federal do Ceará; \\ 2UnB - Universidade de Brasília; \\ 3Fiocruz Ceará.
}

Introduction: Rituximab is a monoclonal antibody used to treat diseases in which the depletion of B cells might be beneficial, such as Non-Hodgkin Lymphoma and rheumatoid arthritis. Rituximab's mechanism of action is intrinsically linked to its capacity to interact with the CD20 antigen, a membrane protein present on mature B cells, making protein engineering strategies, such as directed evolution, an important tool for altering the antigen binding region of this antibody, improving its affinity. Thereby, fragments such as scFv (single-chain fragment variable) may be used, since they are small and less complex, but retain the amino acids that compose the variable portion of antibodies, responsible for antigen recognition.

Objective: Construction of a library of rituximab's scFv gene and its expression in filamentous phage surface, aiming to mature the affinity of the antibody for the CD20 antigen by phage display approach.

Methodology: The variability of the library was obtained through error-prone PCR (epPCR), in which the components of the polymerase chain reaction were altered in order to insert random mutations in the scFv DNA sequence. Different conditions were tested in order to provide desired mutation rate. The randomly mutated sequences were cloned into $\mathrm{pHEN} 2$ vector, transformed into E. coli to build the library and displayed on phage surface after co-infection with a helper phage (VCSM13). The surface-expressing scFv phage particles were submitted to selection steps, based on the interaction of the mutants to a synthetic peptide, corresponding to the native antibody's epitope. After two rounds of selection with increasing astringent conditions (successive washes to remove non- and low-binding phages), sequences were submitted to DNA sequencing.

Results: The chosen epPCR condition included a $0.3 \mathrm{mM} \mathrm{MnCl} 2,7 \mathrm{mM} \mathrm{MgCl} 2,0.2 \mathrm{mM}$ dATP and dGTP, and $1 \mathrm{mM} \mathrm{dCTP}$ and dTTP, added to a standard reaction. The constructed library, of 1.4 x 105 clones, was analyzed by DNA sequencing. Our library showed a DNA mutation rate in the 726 -bp sequence of $0.56 \%( \pm 0.24)$ and $1.07 \%( \pm 0.76)$ mutation rate at protein level (242 aa), after analysis of twelve random sequences. The mutations were distributed along all the sequence, including framework and CDR regions. All rounds had a phage input of 1010 PFU and 103 phage output.

Conclusion: The constructed library has enough size to perform affinity maturation experiments, with moderate variability, with mutants with up to 6 mutations at protein level. The resulting phages were submitted to PCR with scFv-specific primers and are currently under sequencing analysis. It is expected that the selected scFvs harbor point mutations that may play an important role in affinity maturation. If confirmed, it is possible to improve their therapeutic potential and these mutants can be further exploited as innovative biopharmaceuticals with different potential use, such as bispecific molecules and chimeric antigen receptors (CAR).

Keywords: Phage Display; Directed Evolution; scFv 